This is a preprint or reprint of a paper intended for presentation at a conference. Because changes may be made before formal

publication, this is made available with the understanding that it will

not be cited or reproduced without the permission of the author.

\title{
A Low Cost Traveling Wave Tube for Wireless Communications
}

\author{
Bernard Kenneth Vancil'1, and Edwin G. Wintucky² \\ 'FDE Incorporated, Beaverton, OR 97007, USA ${ }^{2}$ NASA Glenn Research Center, Cleveland, OH 44135, USA
}

\begin{abstract}
Demand for high data rate wireless communications is pushing up amplifier power, bandwidth and frequency requirements. Some systems are using vacuum electron devices again because solid-state power amplifiers are not able to efficiently meet the new requirements. The traveling wave tube is the VED of choice because of its excellent broadband capability as well as high power efficiency and frequency. But TWTs are very expensive on a per watt basis below about 200 watts of output power. We propose a new traveling wave tube that utilizes cathode ray tube construction technology and electrostatic focusing. We believe the tube can be built in quantity for under $\$ 1,000$ each. We discuss several traveling wave tube slow wave circuits that lend themselves to the new construction. We will present modeling results and data on prototype devices.
\end{abstract}

\section{INTRODUCTION}

Traveling wave tubes have primarily been used in military and satellite systems. Rarely have they been needed in commercial systems. Where high power and high frequency were required, bandwidth requirements have been minimal. Thus klystrons or magnetrons could be used. The ubiquitous microwave oven is a case in point. Single frequency magnetrons with kilowatts of output can be built for about $\$ 20$.

Other commercial high power applications usually did not require high frequency. Radio and television transmitters come to mind. They can utilize conventional power tubes: triodes, tetrodes and pentodes. Of course, there are many commercial applications where high frequency and bandwidth are required, but in most cases, power required was minimal - under about 25 watts. Solid-state amplifiers can satisfy these requirements. Solid-state amplifiers are compact, light, long lived, have instant turn-on and are very inexpensive. They are somewhat inefficient and sometimes suffer from catastrophic failure. Examples include radio and television receivers and cell phones.

Now a commercial application has appeared that requires all things - higher power, significant bandwidth, high frequency, high efficiency, long life - all the things that heretofore were found only in military and aerospace systems. This application is high data rate wireless communications. An example would be high-speed internet transmissions. After much study it seems that only traveling wave tubes are capable of meeting the new requirement. But TWTs as currently constructed are very expensive - over $\$ 10,000$ in some cases. The problem arises because they are linear beam devices. In other words the electron beam must propagate over a long distance while it interacts with an RF circuit. This beam must have considerable power. A TWT operating in a linear regime and producing 100 watts of RF power may need an electron beam of 1500 watts. Focusing a beam of this power is a daunting task, especially if the voltage is to be kept reasonably low. Up till now it has taken powerful magnets to focus it. This is the primary reason TWTs are expensive. The 100 or more samarium cobalt magnets are a significant cost driver. They can add $\$ 1,000$ to the cost of a TWT. Moreover they constitute about one third of the weight. Also, after the beam has interacted with the RF circuit, its power must be recovered, if reasonable efficiency is to be maintained. This involves a complicated series of collectors - each with its own power supply. Also present TWTs are made out of metal and ceramic with many vacuum tight brazed joints. This also raises the cost.

Vacuum electron devices need not be expensive - for instance the microwave oven magnetron for $\$ 20$ and the cathode ray tube at about $\$ 20$. The cathode ray tube is a good device to study, because it too has a long well focused beam. Also it has a glass envelope, and its long train of metal parts - many at different voltages - are held in relative alignment with glass rods. If a TWT could be built in the same way, its cost would be much lower.

But to use the "rodded" structure and glass envelope magnets must be eliminated. In order to focus effectively, magnets must be very close to the electron beam, or have iron pole pieces on each side of the magnet to carry the magnetic field close to the beam. This is not practical when a glass envelope is used. The magnets would have to be on the outside of the envelope, which is too far away. The answer is to use electrostatic focusing of the electron beam.

This work was supported by a Phase II Small Business Innovation Research Grant from NASA Glenn Research Center, Cleveland, $\mathrm{OH}$. 


\section{TYPES OF SLOW WAVE CIRCUITS INVESTIGATED}

Types of RF slow wave interaction circuits for which electrostatic focusing is feasible include 1) coupled cavity 2) ladder or comb, 3) double helix, 4) double ring-loop.

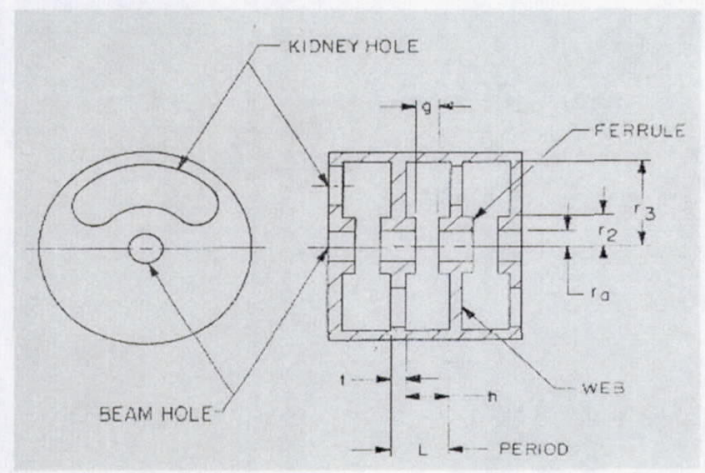

Coupled cavity cross sectional drawing

Fig. 1. Coupled cavity circuit.

1) A coupled cavity circuit is shown in Figure 1. Individual cavities must be isolated electrically from each other via polymide shims. This material has dielectric strength of up to 6,000 volts per mil. It also can stand temperatures in excess of $400^{\circ} \mathrm{C}$, which are necessary in tube processing. We have built a prototype device that produced a focused beam with greater than $95 \%$ transmittance using voltages of alternating polarity between adjacent cavities. We also have done extensive $\mathrm{RF}$ modeling and testing on this circuit. The coupled cavity circuit can be used between $8 \mathrm{GHz}$ and perhaps 40 $\mathrm{GHz}$. Below $8 \mathrm{GHz}$ the circuit becomes too large to be built on standard CRT manufacturing equipment.

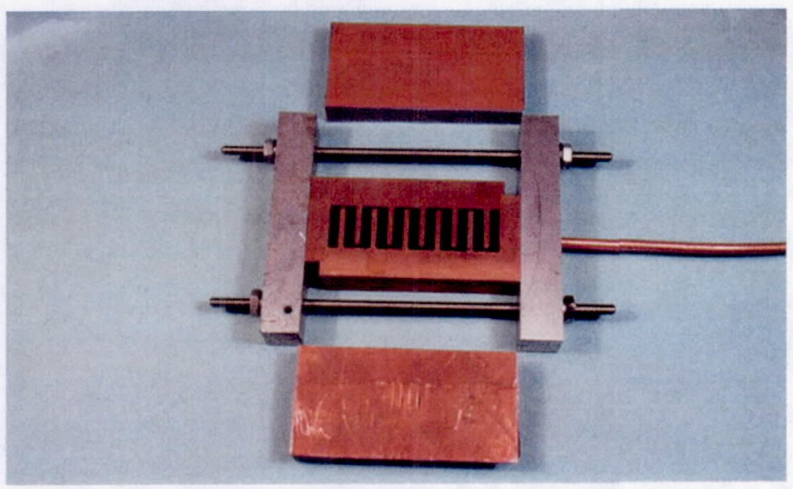

Photo of ladder circuit

Fig. 2. Prototype device using the ladder circuit.
2) We have built a prototype device using the ladder circuit shown in Figure 2, and have done extensive modeling of both RF and electrostatic focusing characteristics. It too focused with better than $95 \%$ transmittance. Numerous drawbacks were discovered, but the most serious was the very low beam interaction impedance.

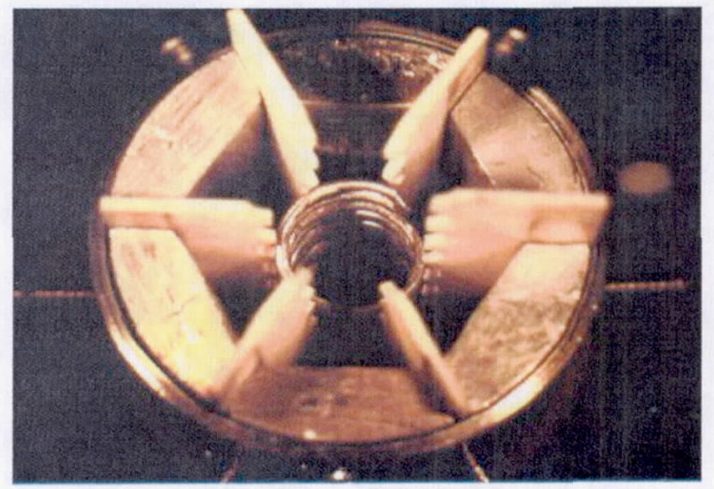

Photo of supported double helix

Fig. 3. Helices in their ceramic support structure.

3) Two intermeshed helices can generate focusing fields if a voltage is applied between them. In the beam tester we built each helix was supported by 3 ceramic plates, .020 inches thick and having teeth which contact and hold its helix wires but not the adjacent ones. Figure 3 shows the helices in their support structure. Extensive modeling was done on the RF characteristics of the double helix. It was found to have higher interaction impedance than the single helix, but it also has a higher tendency for backward wave oscillations. Also the modeling of the electron beam focusing indicated a beam-filling factor of $70 \%$, not as good as the coupled cavity or ladder structures. The prototype device had beam transmittance greater than $93 \%$. It has the advantage that a helix at $2.5 \mathrm{GHz}$ is only .250 inches in diameter. Thus the tube can be made on standard CRT production equipment.

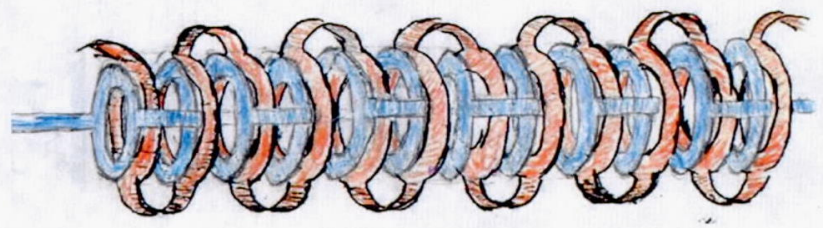

Perspective drawing of Ring Loop unsupported

Fig. 4. Drawing of Double Ring Loop circuit. 
4) A sketch of the double ring-loop circuit is shown in Figure 4. No modeling tools are available to calculate RF characteristics. However, we have built prototype circuits and have measured interaction impedance and phase velocity. We achieved a velocity similar to the double helix and impedance is even higher than the double helix. This circuit is also very compact - only .250 inches in diameter for $2.5 \mathrm{GHz}$ operation. Thus a tube containing it can be built on standard CRT production equipment. This circuit has many other advantages - it is cheap to build, there are no backward wave oscillations and severs and couplers are easy to integrate into the structure. Modeling indicates that it focuses at least as well as the double helix. It is the best candidate for low frequency operation.

\section{FEASIBILITY OF ELECTROSTATIC FOCUSING}

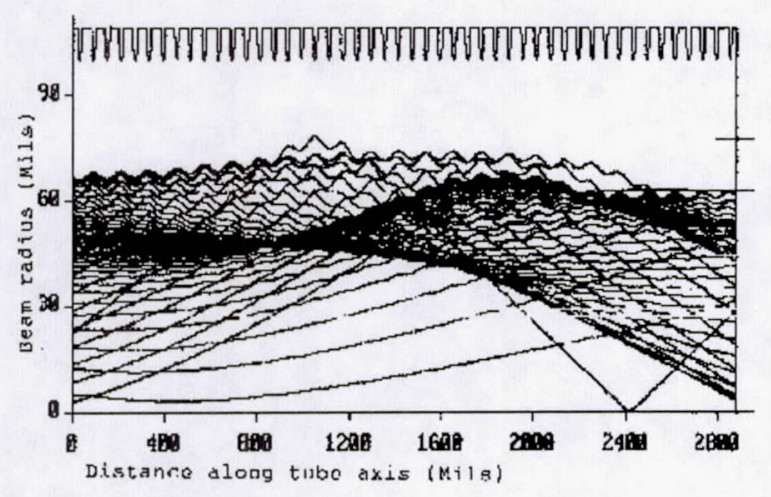

\section{Beam trajectory plot}

Fig. 5. Beam trajectory plot of alternating polarity rings.

Figure 5 shows beam trajectory plots for a series of flat washers placed .040 inches apart. The applied voltage alternates in polarity from washer to washer. This geometry is a good approximation for the ring-loop circuit and a fair approximation for the double helix circuit. The model fails to take into account the rotating transverse electric field of the double helix. This in turn results in a screw like motion of the electron beam. However, analytical calculations indicate that it is a small effect. Moreover, we have built both ring loop and double helix beam testers with perveance greater than .2 micro-pervs that yielded greater than $93 \%$ transmission.

Figure 6 shows beam-filling factor for different perveance beams vs. $\mathrm{DV} / \mathrm{V}$, which is the voltage difference between adjacent washers divided by average beam voltage. It is clear that a filling factor of about $70 \%$ is achievable with a beam perveance of .24 micro-pervs.
This beam corresponds to a beam power of 1600 watts at 8500 volts and a current of $188 \mathrm{ma}$. Saturated RF output is estimated at 400 watts. Reducing this by $6 \mathrm{~dB}$ to linearize tube response will allow us to build a communications tube with output power of 100 watts at $2.5 \mathrm{GHz}$

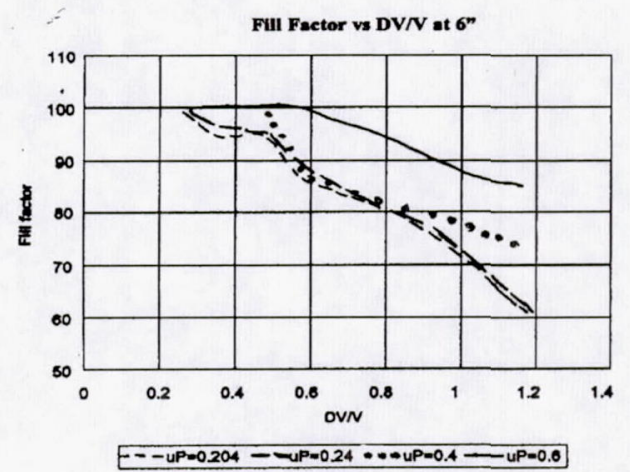

Beam filling factor

Fig. 6. Beam filling factor vs. DV/V for various beam perveances for alternating polarity rings.

It should be remembered that the diameters of the helices or the rings (in ring-loop case) are dictated by RF considerations. Focusing must be adjusted accordingly. The calculated filling factor of about $70 \%$ is not unexpected. In both the ring-loop and double helix cases the requirement to hit the $2.5 \mathrm{GHz}$ telecommunications frequency requires loops that are about .25 inches in diameter. Meanwhile spacings between loops are about .040 inches. This yields electrostatic fields that are small, near the axis but very strong near the loops. Space charge tends to expand the beam to its maximum diameter. Net effect is a beam that is somewhat hollow and resides mostly at the $60-70 \%$ position. The Figure 5 trajectory plot shows how the uniform paraxial beam "hollows" out.

Coupled cavity circuits give the maximum flexibility as to focusing design. The tunnel diameter can be changed to optimize focusing without upsetting wave propagation parameters. This was not the case for either ring-loop or double helix.

Thus the tunnel radius can be made less than the spacing between cavity walls. This results in good focusing throughout the cross section of the beam. The appropriate model both for calculating and building a beam tester is a series of flat wafers with center holes disposed perpendicularly to a central axis. Figure 7 shows a photograph of a section of such a flat wafer design. Notice the glass rods used for holding wafers in relative alignment. 


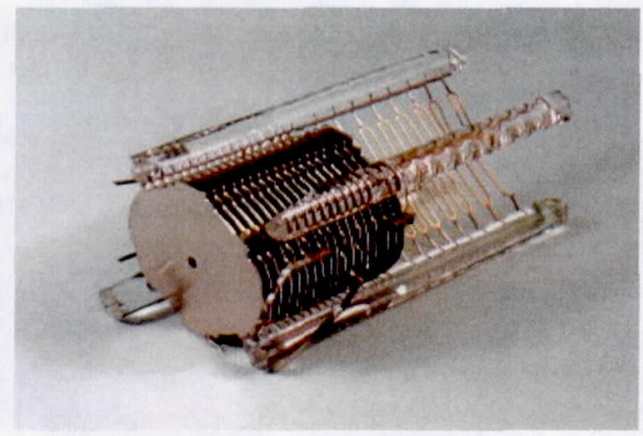

Flat Wafer Focusing Structure

Fig. 7 Photo of flat wafer focusing structure with glass rod fastening.

\section{OTHER CONSIDERATIONS}

Figure 8 compares threshold currents for backward wave oscillation between a conventional helix tube and the electrostatically focused double helix tube. The current is plotted against circuit length. The double helix is more prone to BWO Also the larger the beam the lower the threshold. The modeling results indicate that beam filling factors are larger in electrostatically focused tubes - about $60-70 \%$ vs. the $50-60 \%$ in magnetically focused tubes. This makes the double helix circuit even more prone to BWO. There are methods for suppressing BWO, such as the resonant loss technique, but they are costly.

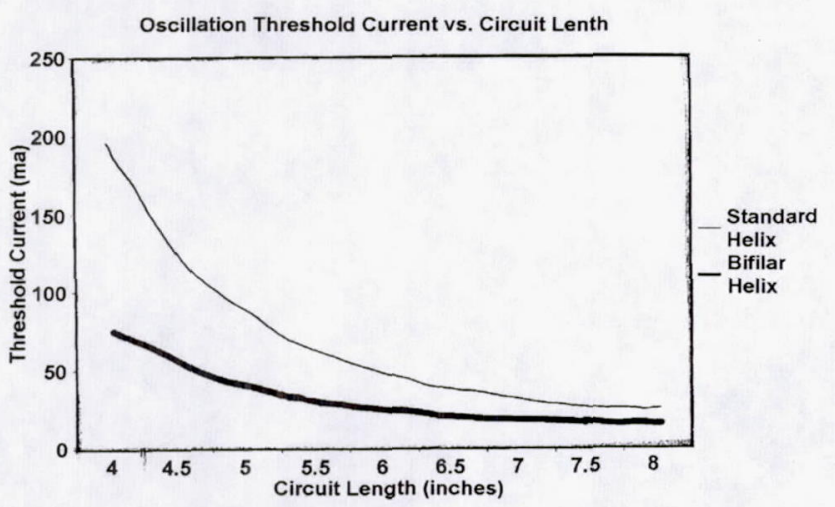

BWO Currents vs. Circuit length

Fig. 8 BWO onset currents vs. circuit length for single and double helix.

It seems that a better approach is to use the ring-loop circuit for low frequency applications. It has no BWO and it is very cheap to build. It has less power capability than ordinary helix tubes because the support ceramics are not as intimate with the circuit. However, our unique mounting system partially overcomes this problem. Also our requirements are only $100-200$ watts of $\mathrm{CW}$ output power. Of great value is its compactness.

Above frequencies of $20 \mathrm{GHz}$ focusing becomes more difficult for ring loop circuits, but easier for ladder circuits and coupled cavity circuits. The coupled cavity circuit has far higher interaction impedance than the ladder circuit. This and other reasons caused us to discard the ladder circuit as a candidate. Also our coupled cavity approach is very close to ordinary coupled cavity circuits in terms of coupler design, dispersion characteristics and interaction impedance. The couplers can be at ground potential which eliminates the need for a complicated DC block. The main difference is that each successive cavity is isolated electrically via a kapton shim. The kapton is only .003 inches thick which permits a very narrow gap between cavities. Thus RF leakage is very small.

\section{CONCLUSIONS}

All the circuits listed in II provide reasonable focusing, but not all of them provide sufficient interaction to give 40 $\mathrm{dBs}$ of gain in a reasonable length. The ladder circuit is an example of one that doesn't. Computer simulation indicates very low interaction impedance. The double helix provides high interaction - higher than a single helix. But backward wave oscillations are also much increased. Without sophisticated resonant loss techniques modeling indicates that this circuit will be unsuitable. Thus we are left with the ring-loop circuit and the coupled cavity circuit. Coupled cavity lends itself mainly to high frequency applications $8 \mathrm{GHz}$ or above. Below 8 the cavity size becomes too large to permit CRT construction techniques - at least on standard production equipment. The focusing is superior because the focusing elements are wafers, with very favorable pitch to tunnel radius ratios. Also focusing geometry can be adjusted without unduly upsetting the RF slow wave circuit performance. Coupled cavity will cost more than ring-loop.

As for ring loop, the loops can be adjusted in length to change wave velocity, while spacing of the rings is adjusted for optimum focusing. The loops provide an extra adjustment "knob", not available on the double helix. Also the ring loop geometry has very high interaction with the beam, which shortens overall tube length. This can only help beam transmittance. Most of all, the ring loop does not support backward wave oscillations. A further advantage is that it is cheap to make! We are currently building a coupled cavity TWT at $10 \mathrm{GHz}$ and a ring-loop TWT at $2.5 \mathrm{GHz}$ 